\title{
Baryon number transport at LHC energies with the ALICE experiment
}

\section{Panos Christakoglou for the ALICE Collaboration*t}

NIKHEF - Utrecht University

E-mail: Panos.Christakoglouecern.ch

Particle yields along with the ratios of particle production in hadronic interactions are important indicators of the collision dynamics. In particular, the detailed analysis of the baryon spectra as well as that of $\bar{p} / p$ and $\bar{\Lambda} / \Lambda$ ratios are of great importance since they allow to determine the carrier of the baryon number (BN). In this paper, the expected performance of the ALICE detector setup regarding the baryon spectra, the rapidity and transverse momentum dependence of the $\bar{p} / p$ and $\bar{\Lambda} / \Lambda$ ratios will be discussed.

The 2009 Europhysics Conference on High Energy Physics,

July 16 - 222009

Krakow, Poland

\footnotetext{
* Speaker.

$\dagger$ The author ackwnoledges the valuable contribution of: Helene Ricaud, Marek Chojnacki, Boris Hippolyte, Alexander Kalweit, Mikolaj Krzewicki and Antonin Maire.
} 


\section{Introduction}

Baryon number transport is one of the key observables that has been debated for some time in connection with the ongoing search for nonlinear dynamical phenomena in nuclear reactions. Based on the quark-gluon string model (QGSM) [1], the baryon number cannot be transported over large rapidity gaps, resulting into a zero net-baryon density at mid-rapidity. This prediction does not properly describe the latest experimental results [2]. Models based on the String Junction (SJ) configuration [3, 4, 5], expect significant baryon/anti-baryon asymmetry even at LHC energies, where the rapidity gap between the beam fragmentation and the mid-rapidity regions will be significantly enhanced.

\section{Baryon transport measurements with ALICE}

Experimentally, information on the baryon transport can be obtained by the measurement of the $\bar{p} / p$ and the $\bar{\Lambda} / \Lambda$ ratios. These will be among the first measurements that the ALICE experiment can perform [6].

Three different analysis scenarios are implemented for the proton case [7]:

- Stand-alone Time Projection Chamber (TPC) analysis: We rely on the first iteration of the tracking where the track is extrapolated from the outer to the inner TPC radius[6]. In parallel, the primary vertex is calculated from the TPC reconstructed tracks. The particle identification information is extracted from the TPC $d E / d x$ measurement.

- Hybrid TPC analysis: Both the tracking and the particle identification is based on the previous approach. The primary vertex is calculated from the Silicon Pixel Detector (SPD) [6]. In addition, in order to better constrain the track, associated Inner Tracking System (ITS) clusters are requested. This reduces the contamination from secondary particles to $2 \%$ [7].

- Global tracking: The tracking and the particle identification relies on the combination of the central barrel detectors. The primary vertex is chosen to be the most accurate among the one calculated from the TPC, the SPD and the tracks produced from the global tracking.

The raw spectra for both $p$ and $\bar{p}$ are corrected taking into account both the absorption and the feed-down corrections. Figure 1 shows the $\eta$ dependence of the absorption correction factors for $p$ (left plot) and $\bar{p}$ (right plot). For the latter the corresponding correction factor is $15 \%$ higher than the one for the protons. Figure 2 shows the $\eta$ dependence of the feed-down influence for the two particles for the different analysis modes. The stand-alone TPC approach results into a correction factor of $\approx 11 \%$, whereas the corresponding values for the other two modes are $\approx 2 \%$.

The systematic uncertainties are estimated taking into consideration the uncertainties related to the estimation of the interaction cross-section, to the determination of the material budget, to the influence of pile-up and beam-gas events [7]. The resulting systematic uncertainty is calculated to be close to $1 \%$ [7]. Further studies are in progress to better estimate the systematic error.

The measurement of the $\bar{\Lambda} / \Lambda$ will also be part of the first ALICE measurements. Using the central barrel detectors we are able to detect the hyperons' decay into charged particles [6]. Selecting a clean sample is achieved by applying some selection criteria based on the topology of the 

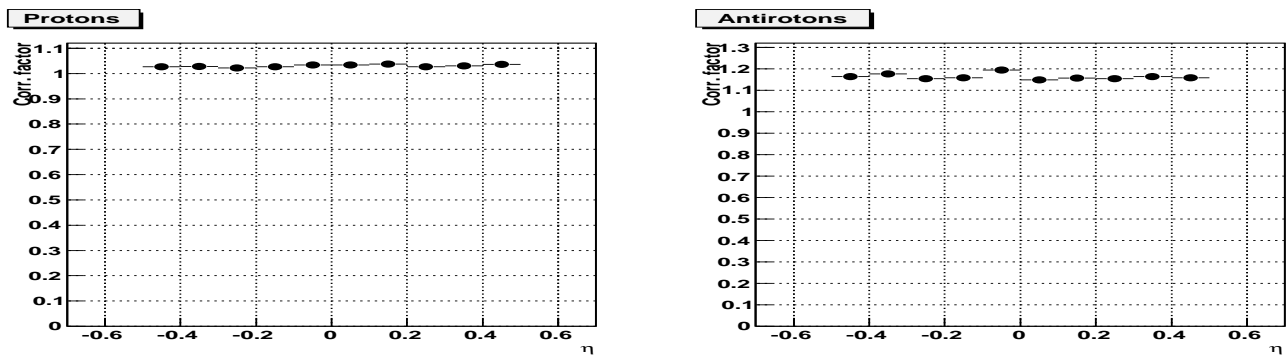

Figure 1: The $\eta$ dependence of the absorption corrections for $p$ (left plot) and $\bar{p}$ (right plot).
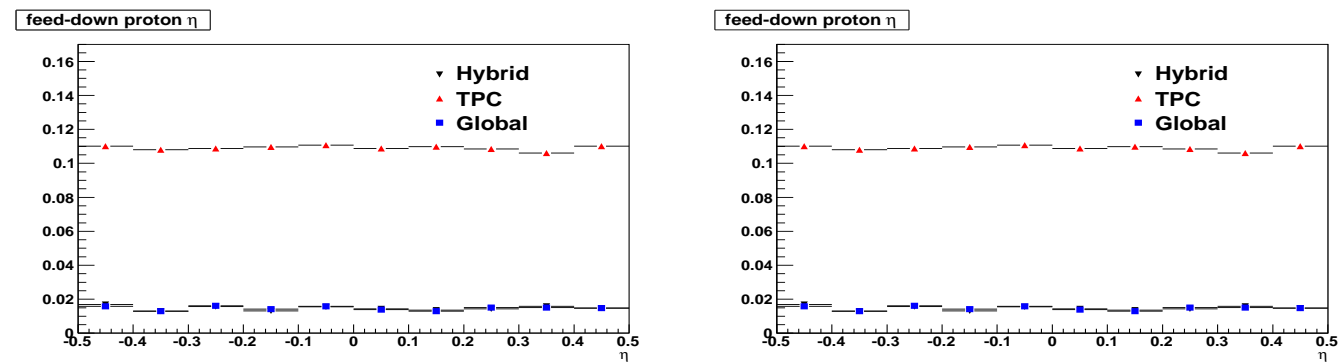

Figure 2: The $\eta$ dependence of the feed-down corrections for $p$ (left plots) and $\bar{p}$ (right plot) for the different analysis modes.

decay. Two V0 finder algorithms are implemented during reconstruction which allow us to compare directly the results from both methods. The analysis of $500 \mathrm{~K}$ minimum-bias $\mathrm{p}+\mathrm{p}$ events, will be sufficient to have a $p_{T}$ range up to $5 \mathrm{GeV} / \mathrm{c}$.

\section{Summary}

In conclusion, the measurement of the $\bar{p} / p$ and $\bar{\Lambda} / \Lambda$ will be among the first measurements of the ALICE experiment. ALICE is well suited and ready for these studies.

\section{References}

[1] G. Cohen-Tannoudji, A.E. Hssouni, J. Kalinowski, R. Peschanski, Phys. Rev. D19, (1979) 3397;

A.B. Kaidalov, Phys. Lett. B52, (1982) 459.

[2] C. Adler et al. [STAR Collaboration], Phys. Rev. Lett. 86, (2001) 4778;

I.G. Bearden et al. [BRAHMS Collaboration], Phys. Rev. Lett. 87, (2001) 112305;

B.B. Back et al. [PHOBOS Collaboration], Phys. Rev. C71, (2005) 021901.

[3] G.C. Rossi, G. Veneziano, Nucl. Phys. B123, (1977) 507.

[4] B.Z. Kopeliovich, B.G. Zakharov, Phys. Lett. B211, (1988) 221.

[5] C. Merino et al., Eur.Phys.J. C54, (2008) 577.

[6] B. Alessandro et al. [ALICE Collaboration], J. Phys. G30, (2004) 1517;

B. Alessandro et al. [ALICE Collaboration], J. Phys. G32, (2006) 1295.

[7] P. Christakoglou, M. Oldenburg, ALICE-INT-2008-010 v.1;

P. Christakoglou, M. Krzewicki, ALICE Internal Note to be submitted. 